\title{
FPGA Device and Architecture Evaluation Considering Process Variations
}

\author{
Ho-Yan Wong, Lerong Cheng, Yan Lin, Lei He \\ Electrical Engineering Department \\ University of California, Los Angeles \\ \{phoebe, lerong, ylin, lhe\}@ee.ucla.edu, http://eda.ee.ucla.edu
}

\begin{abstract}
Process variations in nanometer technologies are becoming an important issue for cutting-edge FPGAs with a multimillion gate capacity. Considering both die-to-die and withindie variations in effective channel length, threshold voltage, and gate oxide thickness, we first develop closed-form models of leakage and timing variations at the FPGA chip level. Experiments show that our models are within $3 \%$ from Monte Carlo simulation, and the leakage and delay variations can be up to $3 \mathrm{X}$ and $1.9 \mathrm{X}$, respectively. We then derive analytical yield models considering both leakage and timing variations, and use such models to evaluate FPGA device and architecture under process variations. Compared to the architecture similar to a commercial FPGA and device setting from ITRS roadmap, device tuning alone improves leakage yield by $39 \%$ and architecture and device co-optimization increases leakage yield by $73 \%$. We also show that LUT size 4 gives the highest leakage yield, LUT size 7 gives the highest timing yield, but LUT size 5 achieves the maximum combined leakage and timing yield. To the best of our knowledge, this is the first in-depth study on FPGA device and architecture co-evaluation considering process variations.
\end{abstract}

\section{INTRODUCTION}

Modern VLSI designs see a large impact from process variation as devices scale down to nanometer technologies. Variability in effective channel length, threshold voltage, and gate oxide thickness incurs uncertainties in both chip performance and power consumption. For example, measured variation in chip-level leakage can be as high as $20 \mathrm{X}$ compared to the nominal value for high performance microprocessors [1]. In addition to meeting the performance constraint under timing variation, dies with excessively large leakage due to such a high variation have to be rejected to meet the given power budget. There have been a few studies on parametric yield estimation considering both timing $[2,3]$ and leakage $[4,5]$ variations in ASICs. However, the parametric yield study for FPGAs is largely unexplored in the literature.

Existing FPGA architecture evaluation has considered performance, area, and power [6, 7, 8, 9]. [10] evaluated new FPGA architectures considering field programmable supply voltage including dual-Vdd and power-gating. A very recent work [11] showed that device and architecture co-optimization is able to obtain the largest improvement in FPGA performance and power efficiency.

*This paper is partially supported by NSF CAREER award CCR-0093273, and NSF grant CCR-0306682. We used computers donated by Intel. Address comments to lhe@ee.ucla.edu.
However, all the evaluation work so far did not consider process variations.

In this paper, we first develop closed-form models of leakage and timing variations at the FPGA chip level with consideration of dieto-die and within-die variations. Experiments show that our models are within 3\% from Monte Carlo simulation, and the leakage and delay variations can be up to $3 \mathrm{X}$ and $1.9 \mathrm{X}$, respectively. In addition, it is also shown that leakage is more sensitive to within-die variation compared to inter-die variation, whereas timing is more sensitive to inter-die variation compared to within-die variation. We then evaluate FPGA device and architecture under process variations. Compared to the architecture similar to a commercial FPGA and device setting from ITRS roadmap, device tuning alone improves leakage yield by $39 \%$ and architecture and device co-optimization increases leakage yield by $73 \%$. We also show that LUT size 4 gives the highest leakage yield, LUT size 7 gives the highest timing yield, but LUT size 5 achieves the maximum combined leakage and timing yield.

The rest of the paper is organized as follows. Section 2 presents background knowledge. Section 3 derives closed-form models for leakage and delay variations. Section 4 develops the leakage and timing yield models. Section 5 analyzes the leakage and timing yield rates, and Section 6 concludes the paper.

\section{BACKGROUND}

We assume the cluster-based island style FPGA same as the existing architecture evaluation work [8]-[11]. A logic block is a cluster of fully connected Basic Logic Elements that consists of one LUT and one flip-flop. The cluster size $N$ and LUT size $K$ are the architectural parameters to be evaluated. For simplicity, we assume a fixed routing architecture same as [11], i.e., fully buffered routing switches and uniform wire segment spanning four logic blocks. We also optimize devices in terms of $V_{d d}$ and $V_{t h}$.

The above architecture and device co-optimization may easily have over hundreds device and architecture combinations. A runtime efficient trace-based estimation tool Ptrace is proposed to handle such co-optimization [11]. For a given benchmark set and a given FPGA architecture, statistical information of switching activity, critical path structure, and circuit element utilization are collected by profiling the placed and routed benchmark circuits. These statistical information is called the trace of the given benchmark set. Then, closed-form formulas are used to compute power and delay based on trace information and device parameters. Ptrace has a high accuracy compared to the detailed verification, where a circuit is placed and routed by VPR [6] and simulated by cycleaccurate power simulation Psim [10].

In this paper, we consider the variation in threshold voltage 
( $\left.V_{t h}\right)$, effective channel length $\left(L_{e f f}\right)$, and gate oxide thickness $\left(T_{o x}\right)$. Similar to [4] where ASIC is assumed, each variation $(\Delta P)$ is decomposed into global (die-to-die) variation $\left(\Delta P_{g}\right)$ and local (within-die) variation $\left(\Delta P_{l}\right)$. We will extend Ptrace to consider the above variations and then perform device and architecture cooptimization with process variations.

\section{LEAKAGE AND TIMING VARIATIONS}

\subsection{Leakage under Variation}

We extend the leakage model in FPGA power and delay estimation framework Ptrace [11] to consider variations. In Ptrace, the total leakage of an FPGA chip is calculated as follows,

$$
I_{\text {chip }}=\sum_{i} N_{i}^{t} \cdot I_{i}
$$

where $N_{i}^{t}$ is the number of FPGA circuit elements in FPGA resource type $i$, i.e., an interconnect switch, buffer, LUT, configuration SRAM cell, or flip-flop, and $I_{i}$ is the leakage of an element. Different sizes of interconnect switches and buffers are considered as different circuit elements.

The leakage current $I_{i}$ of a circuit element $i$ is the sum of the subthreshold and gate leakages:

$$
I_{i}=I_{\text {sub }}+I_{\text {gate }}
$$

Variation in $I_{s u b}$ mainly sources from variation in $L_{e f f}$ and $V_{t h}$. Variation in $I_{g a t e}$ mainly sources from variation in $T_{o x}$.

Different from [4] that models subthreshold leakage and gate leakage separately, we model the total leakage current $I_{i}$ of circuit element in resource type $i$ as follows,

$$
I_{i}=I_{n}(i) \cdot e^{f_{i}\left(\Delta L_{e f f}\right)} \cdot e^{f_{i}\left(\Delta V_{t h}\right)} \cdot e^{f_{i}\left(\Delta T_{o x}\right)}
$$

where $I_{n}(i)$ is the leakage of a circuit element in resource type $i$ in the absence of any variability and $f$ is the function that represents the impact of each type of process variation on leakage. The interdependency between these functions has been shown to be negligible in [4]. From SPICE simulation, we find that it is sufficient to express these functions as simple linear functions. To make the presentation simple, we denote $\Delta L_{e f f}, \Delta V_{t h}$, and $\Delta T_{o x}$ as $L, V$, and $T$, respectively. We can express these functions with this simple notation as follows,

$$
f(L)=-c_{i 1} \cdot L \quad f(V)=-c_{i 2} \cdot V \quad f(T)=-c_{i 3} \cdot T
$$

where $c_{i 1}, c_{i 2}, c_{i 3}$ are fitting parameters decided by SPICE simulations. The negative sign in the exponent indicates that the transistors with shorter channel length, lower threshold voltage, and smaller oxide thickness lead to higher leakage current. We rewrite (3) as follows by decomposing $L, V$ and $T$ in to local $\left(L_{l}, V_{l}, T_{l}\right)$ and global $\left(L_{g}, V_{g}, T_{g}\right)$ components.

$$
I_{i}=I_{n}(i) \cdot e^{-\left(c_{i 1} L_{g}+c_{i 2} V_{g}+c_{i 3} T_{g}\right)} \cdot e^{-\left(c_{i 1} L_{l}+c_{i 2} V_{l}+c_{i 3} T_{l}\right)}
$$

To extend the leakage model (1) under variations, we consider that each element has unique local variations but all elements in one die share the same global variations. Both global and local variations are modeled as normal random variables. The leakage distribution of a circuit element is a lognormal distribution. The total leakage is the sum of all lognormals. The state-of-the-art FPGA chip usually has a large number of circuit elements and therefore the relative random variance of the total leakage approaches zero. Same as [4], we apply the Central Limit Theorem and use the mean of the distribution to approximate the distribution of the sum of lognormals. After integration, we can write the expression of the chip-level leakage as the follows,

$$
\begin{aligned}
I_{\text {chip }} & \approx \sum_{i} N_{i}^{t} \cdot E\left[I_{i}\right] \\
& =\sum_{i} N_{i}^{t} S_{i} I_{L_{g}, V_{g}, T_{g}}(i) \\
S_{i} & =e^{\left(c_{i 1} \sigma_{L_{l}}{ }^{2}+c_{i 2} \sigma_{V_{l}}{ }^{2}+c_{i 3} \sigma_{T_{l}}{ }^{2}\right) / 2} \\
I_{L_{g}, V_{g}, T_{g}}(i) & =I_{n}(i) e^{-\left(c_{i 1} L_{g}+c_{i 2} V_{g}+c_{i 3} T_{g}\right)}
\end{aligned}
$$

where $S_{i}$ is the scale factor introduced due to local variability in $L, V$, and $T . I_{L_{g}, V_{g}, T_{g}}(i)$ is the leakage as a function of global variations. $\sigma_{L_{l}}, \sigma_{V_{l}}$ and $\sigma_{T_{l}}$ are the variances of $L_{l}, V_{l}$, and $T_{l}$, respectively.

For an FPGA architecture with power-gating capability, an unused circuit element can be power-gated to reduce leakage power. In this case, Ptrace calculates the total leakage current as follows,

$$
I_{\text {chip }}=\sum_{i} N_{i}^{u} I_{i}+\alpha_{\text {gating }} \sum_{i}\left(N_{i}^{t}-N_{i}^{u}\right) I_{i}
$$

where $N_{i}^{u}$ is the number of used circuit elements in FPGA resource type $i$ and $\alpha_{\text {gating }}$ is the average leakage ratio between a powergated circuit element and a circuit element in normal operation. Same as [11], $1 / 300$ is used for $\alpha_{\text {gating }}$ in this paper. Similar to (6), (7) can be easily extended to consider variations as follows,

$$
I_{\text {chip }} \approx \sum_{i} N_{i}^{u} E\left[I_{i}\right]+\alpha_{\text {gating }} \sum_{i}\left(N_{i}^{t}-N_{i}^{u}\right) E\left[I_{i}\right](8)
$$

where $E\left[I_{i}\right]$ is still defined as in (6).

\subsection{Timing under Variation}

The performance depends on $L_{e f f}, V_{t h}$, and $T_{o x}$, but its variation is primarily affected by $L_{e f f}$ variation[4]. Below we extend the delay model in Ptrace to consider global and local variations of $L_{\text {eff }}$. The structure of the critical path for each benchmark is obtained for timing analysis. The path delay can be calculated as follows,

$$
D=\sum_{i} d_{i}\left(L_{g}, L_{l}\right)
$$

For circuit element $i$ in the path, $d_{i}\left(L_{g}, L_{l}\right)$ is the delay considering global variation $L_{g}$ and local variation $L_{l} . L_{g}$ is the same for all the circuit elements in the critical path. Given $L_{g}$, we evenly sample a few (eleven in this paper) points within range of $\left[L_{g}-\right.$ $\left.3 \sigma_{L_{l}}, L_{g}+3 \sigma_{L_{l}}\right]$. We then perform SPICE simulation to obtain the delay for each circuit element with these variations. As the delay monotonically decreases when $L_{e f f}$ increases, we can directly map the probability of a channel length to the probability of a delay and obtain the delay distribution of a circuit element. We assume that the local channel length variation of each element is independent from each other. Therefore, we can obtain the distribution of the critical path delay for a given $L_{g}$ as follows by convolution operation,

$P D F(D)=P D F\left(d_{1}\right) \otimes P D F\left(d_{2}\right) \otimes \cdots \otimes P D F\left(d_{i}\right) \otimes \cdots \otimes P D F\left(d_{n}\right)$

\section{YIELD MODELS}

\subsection{Leakage Yield}

The leakage yield is calculated on a bin-by-bin basis where each bin corresponds to a specific value $L_{g}$. For a particular bin, the 
value $L_{g}$ is constant. We can rewrite (6) for chip-level leakage current as follows,

$$
\begin{aligned}
I_{\text {chip }} & =\sum_{i} A_{i} \cdot e^{-c_{i 2} V_{g}} \cdot e^{-c_{i 3} T_{g}} \\
A_{i} & =N_{i} I_{n}(i) S_{i} e^{-c_{i 1} L_{g}}
\end{aligned}
$$

where $A_{i}$ is the leakage current for all circuit elements of resource type $i$ at a value of $L_{g}$ and includes the scale factor $S_{i}$ due to the local variability. Let $X_{i}$ be the leakage consumed by the elements of resource type $i$ and it is a lognormal variable. The chip-level leakage current $I_{\text {chip }}$ is the sum of each lognormal variable $X_{i}$ [4] and it can be expressed as follows,

$$
\begin{aligned}
I_{\text {chip }} & =\sum_{i} X_{i} \\
X_{i} & \sim \operatorname{Lognormal}\left(\log \left(A_{i}\right),\left(\left(c_{i 2} \sigma_{V_{g}}\right)^{2}+\left(c_{i 3} \sigma_{T_{g}}\right)^{2}\right)\right)
\end{aligned}
$$

Same as [4], we model $I_{\text {chip }}$, the sum of the lognormal variables $X_{i}$, as another lognormal random variable. The lognormal variable $X_{i}$ shares the same random variables $\sigma_{V_{g}}$ and $\sigma_{T_{g}}$, and therefore these variables are dependent of each other. Considering the dependency, we calculate the mean and variance of the new lognormal $I_{c h i p}$ as follows,

$$
\begin{gathered}
\mu_{I_{\text {chip }}}=\sum_{i}\left\{\exp \left[\log \left(A_{i}\right)+\frac{\left(c_{i 2} \sigma_{V_{g}}\right)^{2}}{2}+\frac{\left(c_{i 3} \sigma_{T_{g}}\right)^{2}}{2}\right]\right\} \\
\sigma_{I_{\text {chip }}{ }^{2}=\sum_{i}}\left\{\exp \left[2 \log \left(A_{i}\right)+\left(c_{i 2} \sigma_{V_{g}}\right)^{2}+\left(c_{i 3} \sigma_{T_{g}}\right)^{2}\right]\right. \\
\left.\cdot\left[\exp \left(c_{i 2}{ }^{2} \sigma_{V_{g}}^{2}+c_{i 3}^{2} \sigma_{T_{g}}^{2}\right)-1\right]\right\} \\
\quad+\sum_{i, j} 2 \operatorname{COV}\left(X_{i}, X_{j}\right)
\end{gathered}
$$

where the mean of $I_{c h i p}, \mu_{I_{c h i p}}$, is the sum of means of $X_{i}$ and the variance of $I_{c h i p}, \sigma_{I_{c h i p}}$, is the sum of variance of $X_{i}$ and the covariance of each pair of $X_{i}$. The covariance is calculated as follows,

$$
\begin{aligned}
\operatorname{COV}\left(X_{i}, X_{j}\right) & =E\left[X_{i} X_{j}\right]-E\left[X_{i}\right] E\left[X_{j}\right] \\
E\left[X_{i} X_{j}\right] & =\exp \left[\log \left(A_{i} A_{j}\right)+\frac{\left(c_{i 2}+c_{j 2}\right)^{2} \sigma_{V_{g}}{ }^{2}}{2}\right. \\
& \left.+\frac{\left.\left(c_{i 3}+c_{j 3}\right)^{2}{\sigma_{T_{g}}}^{2}\right]}{2}\right] \\
E\left[X_{i}\right] & =\exp \left[\log \left(A_{i}\right)+\frac{\left(c_{i 2} \sigma_{V_{g}}\right)^{2}}{2}+\frac{\left(c_{i 3} \sigma_{T_{g}}\right)^{2}}{2}\right]
\end{aligned}
$$

We then use the method from [4] to obtain the mean and variance $\left(\mu_{N, I_{\text {chip }}}, \sigma_{N, I_{\text {chip }}}{ }^{2}\right)$ of the normal random variable corresponding to the lognormal $I_{\text {chip }}$. As the exponential function that relates the lognormal variable $I_{c h i p}$ with the normal variable $I_{N, \text { chip }}$ is a monotone increasing function, the CDF of $I_{\text {chip }}$ can be expressed as follows using the standard expression for the CDF of a lognormal random variable,

$$
\begin{gathered}
\mu_{N, I_{\text {chip }}}=\frac{\log \left[\mu_{I_{\text {chip }}}{ }^{4} /\left(\mu_{I_{\text {chip }}}{ }^{2}+\sigma_{I_{\text {chip }}}{ }^{2}\right)\right]}{2} \\
\sigma_{N, I_{\text {chip }}}{ }^{2}=\log \left[1+\left(\sigma_{I_{\text {chip }}}{ }^{2} / \mu_{I_{\text {chip }}}{ }^{2}\right)\right] \\
Y_{\text {leak }}\left(I_{\text {chip } \mid L_{g}}\right)=C D F\left(I_{\text {chip }}\right) \\
=\frac{1}{2}\left[1+\operatorname{erf}\left(\frac{\log \left(I_{\text {chip }}\right)-\mu_{N, I_{\text {chip }}}}{\sqrt{2} \sigma_{N, I_{\text {chip }}}}\right)\right]
\end{gathered}
$$

where $\operatorname{erf}()$ is the error function. Given a leakage limit $I_{c u t}$ for $I_{\text {chip }},\left[C D F\left(I_{\text {cut }}\right) \times 100 \%\right]$ gives the leakage yield rate $Y_{\text {leak }}\left(I_{\text {cut }} \mid L_{g}\right)$, i.e., the percentage of FPGA chips that is smaller than $I_{c u t}$ in a particular $L_{g}$ bin. Similarly, the yield for the FPGA chip with power-gating capability can be easily calculated using (8).

\subsection{Timing Yield}

The timing yield is again calculated on a bin-by-bin basis where each bin corresponds to a specific value $L_{g}$. We further consider local variation of channel length in timing yield analysis. Given the global channel length variation $L_{g}$, (10) gives the PDF of the critical path delay $D$ of the circuit. We can obtain the CDF of delay, $C D F\left(D \mid L_{g}\right)$, by integrating for a given $L_{g}$. Given a cutoff delay ( $\left.D_{\text {cut }}\right)$ and $L_{g}, C D F\left(D_{\text {cut }} \mid L_{g}\right)$ gives the probability that the path delay is smaller than $D_{\text {cut }}$ considering $L_{e f f}$ variations. However, it is not sufficient to only analyze the original critical path in the absence of process variations. The close-to-be critical paths may become critical considering variations and an FPGA chip that meets the performance requirement should have the delay of all paths no greater than $D_{\text {cut }}$.

We assume that the delay of each path is independent and we can calculate the timing yield for a given $L_{g}$ as follows,

$$
Y_{\text {perf }}\left(D_{\text {cut }} \mid L_{g}\right)=\prod_{i=1}^{n} C D F_{i}\left(D_{\text {cut }} \mid L_{g}\right)
$$

where $C D F_{i}\left(D_{c u t} \mid L_{g}\right)$ gives the probability that the delay of the $i^{\text {th }}$ longest path is no greater than $D_{c u t}$. In this paper, we only consider the ten longest paths, i.e., $n=10$ because the simulation result shows that the ten longest paths have already covered all the paths with a delay larger than $75 \%$ of the critical path delay under the nominal condition. We then integrate $Y_{\text {perf }}\left(D_{\text {cut }} \mid L_{g}\right)$ to calculate the performance yield $Y_{\text {perf }}$ as follows,

$$
Y_{\text {perf }}=\int_{-\infty}^{+\infty} \operatorname{PDF}\left(L_{g}\right) \cdot Y_{\text {perf }}\left(D_{\text {cut }} \mid L_{g}\right) \cdot d L_{g}
$$

\subsection{Leakage and Timing Combined Yield}

To analyze the yield of a lot, we need to consider both leakage and delay limit. Given a specific global variation of channel length $L_{g}$, the leakage variability only depends on the variability of random variable $V_{g}$ and $T_{g}$ as shown in (6), and the timing variability only depends on the variability of random variable $L_{l}$. Therefore, we assume that the leakage yield and timing yield are independent of each other. The yield considering the imposed leakage and timing limit can be calculated as follows,

$$
Y_{\text {com }}=\int_{-\infty}^{+\infty} \operatorname{PDF}\left(L_{g}\right) Y_{\text {leak }}\left(I_{\text {cut }} \mid L_{g}\right) Y_{\text {perf }}\left(D_{\text {cut }} \mid L_{g}\right) \cdot d L_{g}
$$

\section{LEAKAGE AND TIMING YIELD ANALYSIS}

For the total power and leakage power we report the arithmetic mean of $20 \mathrm{MCNC}$ benchmarks within and among three FPGA architecture classes: $H o m o-V_{t}$ is the conventional FPGA using the same and optimized $V_{t}$ for both logic blocks and interconnect; Hetero- $V_{t}$ optimizes $V_{t}$ separately for logic blocks and interconnect; and Homo- $V_{t}+G$ is the same as Homo- $V_{t}$ except that unused logic blocks and interconnect are power-gated as studied in[10]. We assume $10 \%$ of the nominal value as $3 \sigma$ for all the process variations.

\subsection{Leakage Yield}

Figure 1 shows the full chip leakage power simulated by Monte Carlo simulation and $\sigma$, in the presence of inter-die and intra-die variations. Leakage may change significantly due to process variations. When there is a $\pm 3 \sigma$ inter-die variation of $L_{e f f}$, the leakage power has a $3 X$ span. When no variation is present, there is still a $2 X$ span in leakage power due to within-die variation. Clearly, 


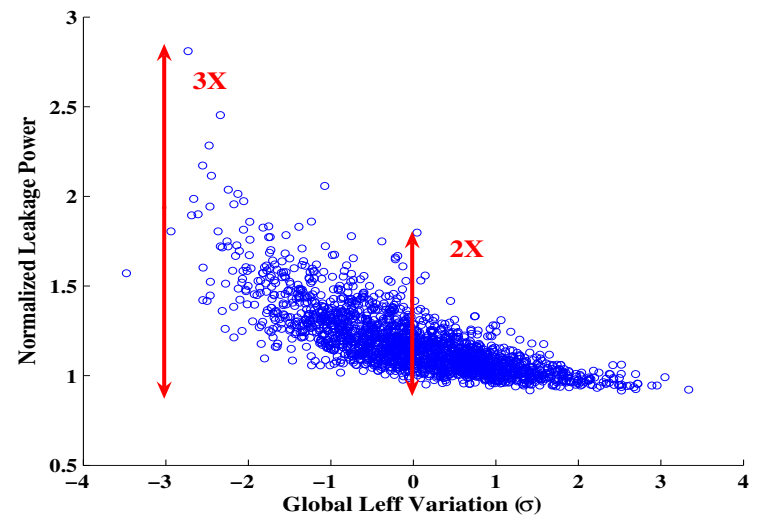

Figure 1: Leakage power of baseline architecture $(\mathrm{N}=8, \mathrm{~K}=4)$ with ITRS device setting under intradie and inter-die variations.

leakage is more sensitive to within-die variation compared to interdie variation. Therefore it is important to consider the impact of process variations on leakage when determining the yield.

We further validate our chip-level analytical model for leakage by Monte Carlo simulation to estimate the full chip leakage power in Table 1, where global variations are all set to $\pm 3 \sigma$, and local variations are set to $0, \pm 1 \sigma$, and $\pm 2 \sigma$. The mean calculated from our analytical method has a less than $3 \%$ difference from the simulation and the standard deviations differed by $1 \%$ of the mean value. In the rest of the paper, we always report the standard deviation as a relative value with respect to the mean and use our analytical model to calculate the yield.

\begin{tabular}{|c|c|c|c|c|c|c|}
\hline \multicolumn{3}{|c|}{ Variations $(\sigma)$} & \multicolumn{2}{c|}{ Mean(W) } & \multicolumn{2}{c|}{ SD(\%) } \\
\hline$\left(L_{g}, L_{l}\right)$ & $\left(V_{g}, V_{l}\right)$ & $\left(T_{g}, T_{l}\right)$ & $M-C$ & Model & $M-C$ & Model \\
\hline$( \pm 3,0)$ & $( \pm 3,0)$ & $( \pm 3,0)$ & 1.24 & 1.20 & 14 & 13 \\
\hline$( \pm 3, \pm 1)$ & $( \pm 3, \pm 1)$ & $( \pm 3, \pm 1)$ & 1.41 & 1.37 & 14 & 13 \\
\hline$( \pm 3, \pm 2)$ & $( \pm 3, \pm 2)$ & $( \pm 3, \pm 2)$ & 2.07 & 2.00 & 13 & 12 \\
\hline
\end{tabular}

Table 1: Comparison between analytical variation models and Monte Carlo ( $M-C$ ) simulation.

\subsubsection{Impact of Architecture and Device Tuning}

In this section we consider combinations of device and architecture parameters, called as hyper-architecture (in short, hyperarch). Table 2 shows the yield, mean leakage, and standard deviation from two different device settings, sorted by the yield. Columns 1-4 use ITRS device setting. Our baseline FPGA has $N=8$ and $K=4$, which is the architecture used by Xilinx Virtex-II Pro. Yield is calculated using the nominal leakage of each architecture plus an offset of $30 \%$ of the nominal leakage of baseline architecture, $P_{\text {base }}^{L}$, as the leakage limit. As shown in column 1 of Table 2, the yield ranges from $24 \%$ to $70 \%$, which shows that architecture tuning has a significant impact on the yield. Among all architectures, $N=6$ and $K=5$ gives the maximum yield, which is $12 \%$ higher than the baseline. The yield is affected by both the mean and variance. When the mean leakage is close to the leakage limit, the variance gains importance in determining the yield. However, when the mean is not close to the limit, the variance does not have that much impact on the yield. In this case, the lower the mean leakage is, the higher the yield is (see columns $5-8$ ). It is also noticeable that larger LUT sizes have larger mean leakage, thus yield becomes smaller.

\begin{tabular}{|c|c|c|c|c|c|c|c|}
\hline 1 & 2 & 3 & 4 & 5 & 6 & 7 & 8 \\
\hline \multicolumn{3}{|c|}{ ITRS Vdd0.80V/V $/ V_{t} 0.20 \mathrm{~V}$} & \multicolumn{3}{|c|}{ Min ED Vdd0.90V $/ V_{t} 0.30 \mathrm{~V}$} \\
\hline $\begin{array}{c}\text { Y } \\
(\%)\end{array}$ & $\begin{array}{c}\text { Mean } \\
(\mathrm{W})\end{array}$ & $\begin{array}{c}\text { SD } \\
(\%)\end{array}$ & $(\mathrm{N}, \mathrm{K})$ & $\begin{array}{c}\text { Y } \\
(\%)\end{array}$ & $\begin{array}{c}\text { Mean } \\
(\mathrm{W})\end{array}$ & $\begin{array}{c}\text { SD } \\
(\%)\end{array}$ & $(\mathrm{N}, \mathrm{K})$ \\
\hline 70 & 0.40 & 39 & $(6,5)$ & 97 & 0.07 & 48 & $(6,4)$ \\
\hline 68 & 0.50 & 40 & $(8,3)$ & 97 & 0.08 & 48 & $(8,4)$ \\
\hline 64 & 0.58 & 39 & $(10,3)$ & 96 & 0.08 & 48 & $(10,4)$ \\
\hline 61 & 0.55 & 38 & $(12,3)$ & 96 & 0.08 & 49 & $(6,5)$ \\
\hline 60 & 0.43 & 64 & $(6,4)$ & 94 & 0.10 & 48 & $(8,3)$ \\
\hline 58 & 0.45 & 63 & $(8,4)$ & 93 & 0.12 & 48 & $(10,3)$ \\
\hline 55 & 0.47 & 62 & $(10,4)$ & 92 & 0.11 & 48 & $(12,3)$ \\
\hline 43 & 0.55 & 34 & $(8,5)$ & 89 & 0.11 & 49 & $(12,4)$ \\
\hline 43 & 0.56 & 34 & $(10,5)$ & 88 & 0.11 & 49 & $(8,5)$ \\
\hline 42 & 0.60 & 34 & $(12,5)$ & 87 & 0.11 & 49 & $(10,5)$ \\
\hline 40 & 0.58 & 37 & $(3,6)$ & 87 & 0.12 & 48 & $(3,6)$ \\
\hline 39 & 0.62 & 53 & $(12,4)$ & 86 & 0.12 & 49 & $(12,5)$ \\
\hline 37 & 0.71 & 40 & $(8,6)$ & 78 & 0.15 & 49 & $(6,6)$ \\
\hline 37 & 0.71 & 40 & $(6,6)$ & 78 & 0.15 & 49 & $(8,6)$ \\
\hline 37 & 0.78 & 39 & $(10,6)$ & 76 & 0.16 & 49 & $(10,6)$ \\
\hline 36 & 0.82 & 39 & $(12,6)$ & 75 & 0.17 & 49 & $(12,6)$ \\
\hline 26 & 0.92 & 47 & $(6,7)$ & 72 & 0.17 & 49 & $(6,7)$ \\
\hline 25 & 0.98 & 46 & $(8,7)$ & 70 & 0.18 & 49 & $(8,7)$ \\
\hline 25 & 1.32 & 46 & $(10,7)$ & 68 & 0.25 & 49 & $(10,7)$ \\
\hline 24 & 1.22 & 44 & $(12,7)$ & 65 & 0.23 & 49 & $(12,7)$ \\
\hline
\end{tabular}

Table 2: Comparison of Different Device Setting

Device tuning also affects the yield. In Columns $5-8$ of Table 2 , we use a device setting that provides the minimum energy-delay product (minimum product of energy per clock cycle and critical path delay, in short, min-ED) given in [11]. Column 5 shows that optimizing Vdd and $V_{t}$ can increase the yield rate of each architecture by an average of $39 \%$. Therefore, device tuning has a great impact on yield rate and it is important to evaluate different Vdd and $V_{t}$ levels while considering process variations. Comparing the yield of architecture $(12,7)$ in ITRS device setting and architecture $(6,4)$ in Min-ED device setting shows that combining device tuning with architecture tuning can increase the yield by up to $73 \%$. From the Table, architectures with $\mathrm{K}=4$ generally provides the highest yield rate, and they have the minimum area as reported in previous work such as [11]. In the rest of the paper, we will only consider dominant architectures. Dominant architectures are defined as the group of architectures that either has smaller delay or less energy consumption than others [11]. Fig 2 presents the energy and delay tradeoff between dominant architectures assuming $\mathrm{Homo}-V_{t}$ class.

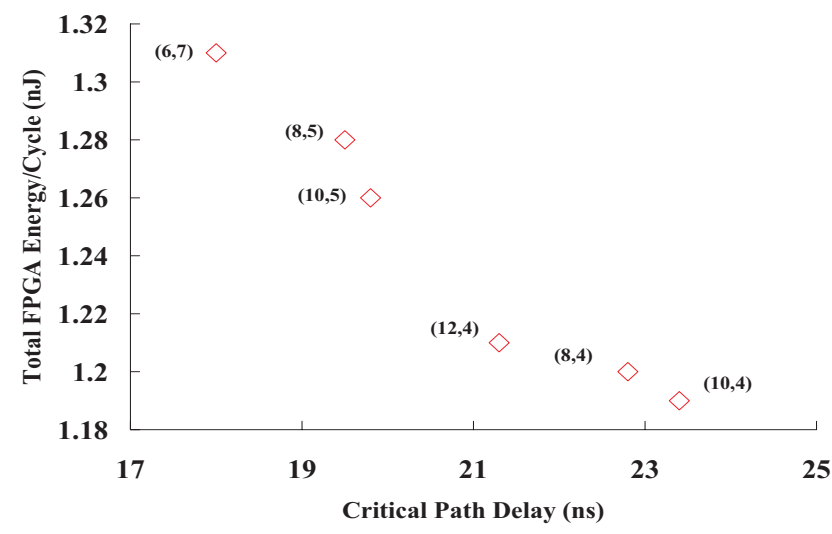

Figure 2: Energy-delay tradeoff among architectures in Homo- $V_{t}$ using min-ED device setting.

\subsubsection{Impact of Heterogeneous- $V_{t}$ and Power-gating}




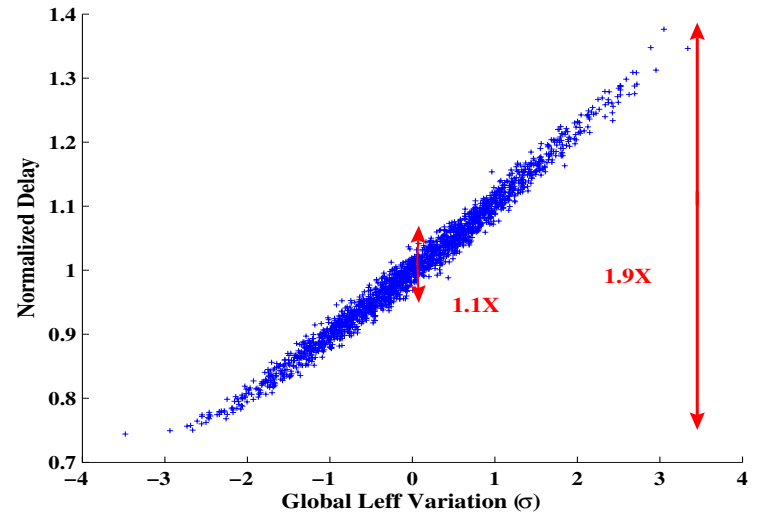

Figure 3: Delay of baseline architecture $(\mathrm{N}=8$, $\mathrm{K}=4$ ) with ITRS device setting under intra-die and inter-die Leff variation .

It has been shown that heterogeneous- $V_{t}$ and power-gating may have great impact on energy delay tradeoff [11]. Here we further consider the impact of heterogeneous- $V_{t}$ on the yield by comparing Homo- $V_{t}$ and Hetero- $V_{t}$ in min-ED device setting. Table 3 shows the results of the dominant architectures in all classes. The average yield for each class is presented in the last row of the table. Comparing the yield of Homo- $V_{t}$ and Hetero- $V_{t}$, we can see that the average yield is improved by $5 \%$ via applying different $V_{t}$ for logic blocks and interconnect. Therefore, introducing heterogeneous- $V_{t}$ could improve yield with no or little area increase (due to an increase in doping well area).

Furthermore, power-gating can be applied to unused FPGA logic blocks and interconnect to reduce leakage power. As only one sleep transistor is used for one logic block, we use a 210X PMOS as the sleep transistor for each logic block. For interconnect, the area overhead associated with sleep transistors is more significant. We therefore use a $2 \mathrm{X}$ PMOS as the sleep transistor for each interconnect switch. Comparing the yield of Homo- $V_{t}$ and Homo- $V_{t}+G$ in Table 3, applying power-gating can improve the yield by $8 \%$. Comparing the yield of Hetero- $V_{t}$ and $H o m o-V_{t}+G$, power-gating can obtain more yield improvement than heterogeneous- $V_{t}$ at the cost of chip-level area overhead between $10 \%$ to $20 \%$. As leakage power can be greatly reduced by power-gating, little benefit can be introduced by applying simultaneous heterogeneous- $V_{t}$ and power-gating, and we will not present the results here. Again, with heterogeneous- $V_{t}$ or power-gating, LUT size $\mathrm{K}=4$ is the best for leakage yield rate.

\subsection{Timing Yield}

For timing yield analysis, we only analyze the delay of the largest MCNC benchmark clma. Similarly, the timing yield is often studied using selected test circuit such as ring oscillator for ASIC in the literature. Figure 3 shows the delay with intra-die and inter-die channel length variation at baseline architecture $(8,4)$ with ITRS device setting. As shown in the figure, there is a $1.9 \mathrm{X}$ span with $\pm 3 \sigma L_{g}$ variation, and a $1.1 X$ span without $L_{g}$ variation. Clearly, delay is more sensitive to inter-die variation than within-die variation. This is because of the independence of local $L_{e f f}$ variation between each element. Therefore the effect of within-die $L_{\text {eff }}$ variation tends to average out when the critical path is long enough.

For timing yield, we discard dies with critical delay larger than the cutoff delay, which is $1.1 X$ of the nominal critical path delay of each architecture. Table 4 shows the delay yield of $\mathrm{Homo}_{-} \mathrm{V}_{t}+G$. One can see from this table that a larger LUT size will give a higher yield rate. This is because a larger LUT size generally gives a smaller mean delay with a shorter critical path (see Fig 2), i.e., smaller number of elements in the path, which leads to a smaller variance. Therefore, a larger LUT size leads to a higher timing yield. As the timing specification may be relaxed for certain applications that are not timing-critical, the cutoff delay may be relaxed in this case. In this table, we also show the yield with the cutoff delay as $1.2 \mathrm{X}$ of the nominal delay. The yield rate under a higher cutoff still has the same trend as that under a lower cutoff. Note that the other architecture classes have similar trends on timing yield.

\begin{tabular}{|c|c|c|c|}
\hline & $\mathrm{Y} 1.1 \mathrm{X}(\%)$ & $\mathrm{Y} 1.2 \mathrm{X}(\%)$ & Mean $(\mathrm{ns})$ \\
\hline$(6,4)$ & 69 & 86 & 39.9 \\
\hline$(8,4)$ & 70 & 86 & 40.7 \\
\hline$(10,4)$ & 69 & 86 & 41.5 \\
\hline$(12,4)$ & 71 & 88 & 38.3 \\
\hline$(6,5)$ & 75 & 91 & 36.4 \\
\hline$(8,5)$ & 74 & 90 & 34.6 \\
\hline$(10,5)$ & 74 & 90 & 34.7 \\
\hline$(6,6)$ & 77 & 93 & 30.8 \\
\hline$(8,6)$ & 78 & 94 & 29.9 \\
\hline$(6,7)$ & 79 & 95 & 27.7 \\
\hline \hline Avg & 75 & 90 & 35.4 \\
\hline
\end{tabular}

Table 4: Timing yield for Homo- $V_{t}+\mathbf{G}$

\subsection{Leakage and Timing Combined Yield}

Figure 4 presents the leakage and delay variation for the baseline case using Monte Carlo simulation with Ptrace. It can be seen that a smaller delay leads to a larger leakage in general. This is because of the inverse correlation between circuit delay and leakage. A device with short channel length has a small delay and consumes large leakage, which may lead to a high leakage. To calculate the leakage and delay combined yield, we set the cutoff leakage as the nominal leakage plus $30 \%$ that of the baseline, while the cutoff delay is $1.2 \mathrm{X}$ of each architecture's nominal delay.

Table 5 presents the combined yield for Homo- $V_{t}$ with ITRS device setting and all classes with min-ED device setting. The area overhead introduced by power-gating is also presented in the table. Comparing $H o m o-V_{t}$ with ITRS device setting and min-ED device setting, the combined yield is improved by $21 \%$. Comparing the classes using min-ED device setting, Hetero- $V_{t}$ has a $3 \%$ higher yield than $H o m o-V_{t}$ due to heterogeneous- $V_{t}$ while $H o m o-V_{t}+G$ has a $8 \%$ higher yield than $H o m o-V_{t}$ due to power-gating. Homo$V_{t}+G$ has the highest combined yield with an average of $16 \%$ area overhead. Device tuning and power-gating improve yield by $29 \%$ comparing Homo- $V_{t}+G$ with min-ED setting to Homo- $V_{t}$ with ITRS setting. This table also shows that architectures with LUT size 5 gives the highest yield within each class. This is because it has both a relatively high leakage yield as well as timing yield.

\section{CONCLUSIONS AND DISCUSSIONS}

In this paper, we have developed efficient models for chip-level leakage variation and system timing variation in FPGAs. Experiments show that our models are within 3\% from Monte Carlo simulation, and the leakage and delay variations can be up to $3 \mathrm{X}$ and $1.9 \mathrm{X}$, respectively. In addition, leakage is more sensitive to within-die variations compared to die-to-die variations, but timing is more sensitive to die-to-die variations. We have shown that architecture and device tuning has a significant impact on FPGA parametric yield rate. LUT size 4 has the highest leakage yield, 7 


\begin{tabular}{|c|c|c|c|c|c|c|c|c|c|c|c|c|c|c|c|c|}
\hline & \multicolumn{5}{|c|}{ Homo- $V_{t}$} & \multicolumn{6}{|c|}{ Hetero- $V_{t}$} & \multicolumn{5}{|c|}{ Homo- $V_{t}+\mathrm{G}$} \\
\hline$(\mathrm{N}, \mathrm{K})$ & $\begin{array}{l}\text { Vdd } \\
(\mathrm{V})\end{array}$ & $\begin{array}{c}V_{t} \\
(\mathrm{~V})\end{array}$ & $\begin{array}{c}\mathrm{Y} \\
(\%)\end{array}$ & $\begin{array}{c}\text { Mean } \\
(\mathrm{W})\end{array}$ & $\begin{array}{l}\text { SD } \\
(\%) \\
\end{array}$ & $\begin{array}{l}\text { Vdd } \\
(\mathrm{V})\end{array}$ & $\begin{array}{l}\mathrm{C} V_{t} \\
(\mathrm{~V})\end{array}$ & $\begin{array}{l}\mathrm{I} V_{t} \\
(\mathrm{~V})\end{array}$ & $\begin{array}{c}\mathrm{Y} \\
(\%)\end{array}$ & $\begin{array}{c}\text { Mean } \\
(\mathrm{W})\end{array}$ & $\begin{array}{l}\text { SD } \\
(\%)\end{array}$ & $\begin{array}{l}\text { Vdd } \\
(\mathrm{V})\end{array}$ & $\begin{array}{c}V_{t} \\
(\mathrm{~V})\end{array}$ & $\begin{array}{c}\mathrm{Y} \\
(\%)\end{array}$ & $\begin{array}{c}\text { Mean } \\
(\mathrm{W})\end{array}$ & $\begin{array}{l}\text { SD } \\
(\%)\end{array}$ \\
\hline$(6,4)$ & 0.90 & 0.30 & 97 & 0.07 & 48 & 0.90 & 0.30 & 0.35 & 99 & 0.06 & 46 & 0.90 & 0.30 & 99 & 0.04 & 48 \\
\hline$(8,4)$ & 0.90 & 0.30 & 97 & 0.08 & 48 & 0.90 & 0.30 & 0.35 & $\overline{99}$ & 0.06 & 46 & 0.90 & 0.30 & $\overline{99}$ & 0.04 & 48 \\
\hline$(10,4)$ & 0.90 & 0.30 & 96 & 0.08 & 48 & 0.90 & 0.30 & 0.35 & 98 & 0.06 & 46 & 0.90 & 0.30 & $\overline{99}$ & 0.04 & 48 \\
\hline$(12,4)$ & 0.90 & 0.30 & 89 & 0.11 & 49 & 0.90 & 0.30 & 0.35 & 96 & 0.08 & 45 & 0.90 & 0.30 & 99 & 0.05 & 48 \\
\hline$(6,5)$ & 0.90 & 0.30 & 96 & 0.08 & 49 & 0.90 & 0.30 & 0.35 & 98 & 0.06 & 46 & 0.90 & 0.30 & 99 & 0.05 & 48 \\
\hline$(8,5)$ & 0.90 & 0.30 & 88 & 0.11 & 49 & 0.90 & 0.30 & 0.35 & $\overline{95}$ & 0.08 & 46 & 0.90 & 0.30 & $\overline{98}$ & 0.05 & 48 \\
\hline$(10,5)$ & 0.90 & 0.30 & 87 & 0.11 & $\overline{49}$ & 0.90 & 0.30 & 0.35 & $\overline{95}$ & 0.08 & 46 & 0.90 & 0.30 & $\overline{98}$ & 0.05 & 48 \\
\hline$(6,6)$ & 0.90 & 0.30 & 78 & 0.15 & 49 & 0.90 & 0.30 & 0.35 & 86 & 0.11 & 46 & 0.90 & 0.30 & 92 & 0.08 & 48 \\
\hline$(8,6)$ & 0.90 & 0.30 & 78 & 0.15 & 49 & 0.90 & 0.30 & 0.35 & 85 & 0.12 & 46 & 0.90 & 0.30 & 91 & 0.08 & 48 \\
\hline$(6,7)$ & 0.90 & 0.30 & 72 & 0.17 & 49 & 0.90 & 0.30 & 0.35 & 77 & 0.14 & 47 & 0.90 & 0.30 & 83 & 0.11 & 48 \\
\hline Avg & 0.90 & 0.30 & 88 & 0.11 & 49 & 0.90 & 0.30 & 0.35 & 93 & 0.08 & 46 & 0.90 & 0.30 & 96 & 0.06 & 48 \\
\hline
\end{tabular}

Table 3: Comparison of leakage yield between classes.

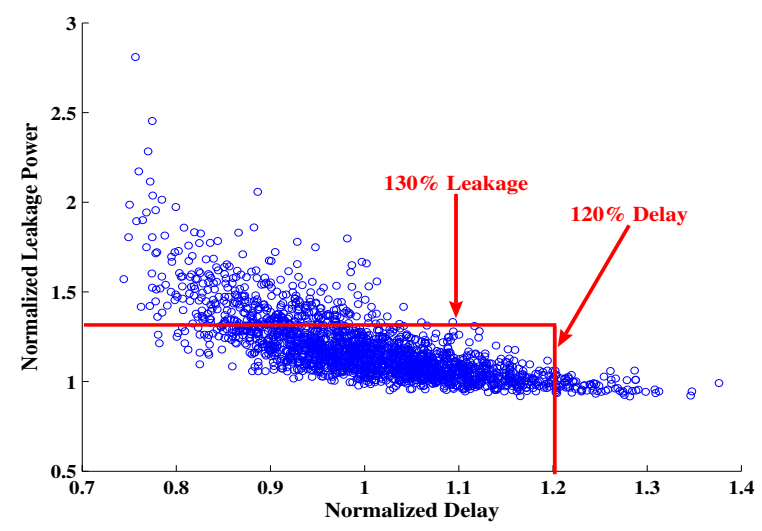

Figure 4: Leakage and delay of baseline architecture $(\mathrm{N}=8, \mathrm{~K}=4)$ with ITRS setting under process variations.

\begin{tabular}{|c|c|c|c|c|c|}
\hline \multirow{3}{*}{$(\mathrm{N}, \mathrm{K})$} & \multirow{3}{*}{$\begin{array}{c}\text { ITRS } \\
\text { Homo- } V_{t} \\
\mathrm{Y}(\%)\end{array}$} & \multicolumn{4}{|c|}{ Min-ED } \\
\hline & & \multirow{2}{*}{$\begin{array}{c}\text { Homo- } V_{t} \\
\mathrm{Y}(\%)\end{array}$} & \multirow{2}{*}{$\begin{array}{c}\text { Hetero- } V_{t} \\
\mathrm{Y}(\%)\end{array}$} & \multicolumn{2}{|c|}{ Homo- $V_{t}+\mathrm{G}$} \\
\hline & & & & $\mathrm{Y}(\%)$ & Area Inc(\%) \\
\hline$(6,4)$ & 71 & 83 & 83 & 86 & 18 \\
\hline$(8,4)$ & 67 & 81 & 81 & 86 & 14 \\
\hline$(10,4)$ & 65 & 81 & 81 & 86 & 17 \\
\hline$(12,4)$ & 48 & 77 & 81 & 87 & 20 \\
\hline$(6,5)$ & 79 & 85 & 84 & 90 & 14 \\
\hline$(8,5)$ & 55 & 81 & 86 & 89 & 15 \\
\hline$(10,5)$ & 55 & 81 & 86 & 89 & 19 \\
\hline$(6,6)$ & 49 & 77 & 82 & 88 & 15 \\
\hline$(8,6)$ & 49 & 75 & 80 & 88 & 16 \\
\hline$(6,7)$ & 45 & 73 & 77 & 86 & 10 \\
\hline Avg & 58 & 79 & 82 & 87 & 16 \\
\hline
\end{tabular}

Table 5: Combined Leakage-delay yield between FPGA Classes.

has the highest timing yield, but LUT size 5 achieves the maximum combined leakage and timing yield. We assume a fixed interconnect structure in this paper, and will study the impact of hierarchical interconnect structure with process variations in the future.

\section{REFERENCES}

[1] S. Borkar, T. Karnik, S. Narendra, J. Tschanz, A. Keshavarzi, and V. De, "Parameter variations and impact on circuits and microarchitecture," in Proc. Design Automation Conf., June 2003.

[2] S. R. Nassif, "Modeling and analysis of manufacturing variations," in Proc. IEEE Custom Integrated Circuits Conf., 2001.
[3] A. Gattiker, S. Nassif, R. Dinakar, and C. Long, "Timing yield estimation from static timing analysis," in International Symposium on Quality of Electronic Design, 2001.

[4] R. Rao, A. Devgan, D. Blaauw, and D. Sylvester, "Parametric yield estimation considering leakage variability," in Proc. Design Automation Conf., June 2004.

[5] S. Zhang, V. Wason, and K. Banerjee, "A probabilistic framework to estimate full-chip subthreshold leakage power distribution considering within-die and die-to-die p-t-v variations," in ISLPED, Aug 2004.

[6] V. Betz, J. Rose, and A. Marquardt, Architecture and $C A D$ for Deep-Submicron FPGAs. Kluwer Academic Publishers, Feb 1999.

[7] V. Betz and J. Rose, "FPGA routing architecture: Segmentation and buffering to optimize speed and density," in Proc. ACM Intl. Symp. Field-Programmable Gate Arrays, Feb 1999.

[8] E. Ahmed and J. Rose, "The effect of LUT and cluster size on deep-submicron FPGA performance and density," in Proc. ACM Intl. Symp. Field-Programmable Gate Arrays, pp. 3-12, Feb 2000.

[9] F. Li, D. Chen, L. He, and J. Cong, "Architecture evaluation for power-efficient FPGAs," in Proc. ACM Intl. Symp. Field-Programmable Gate Arrays, Feb 2003.

[10] Y. Lin, F. Li, and L. He, "Power modeling and architecture evaluation for FPGA with novel circuits for vdd programmability," in Proc. ACM Intl. Symp. Field-Programmable Gate Arrays, February 2005.

[11] L. Cheng, P. Wong, F. Li, Y. Lin, and L. He, "Device and architecture co-optimization for FPGA power reduction," in Proc. Design Automation Conf., June 2005. 